\title{
The double sign of a real division algebra of finite dimension greater than one
}

Erik Darpö and Ernst Dieterich 



\title{
The double sign of a real division algebra of finite dimension greater than one
}

\author{
Erik Darpö and Ernst Dieterich
}

\begin{abstract}
For any real division algebra $A$ of finite dimension greater than one, the signs of the determinants of left multiplication and right multiplication by an element $a \in A \backslash\{0\}$ are shown to form an invariant of $A$, called its double sign. For each $n \in\{2,4,8\}$, the double sign causes the category $\mathscr{D}_{n}$ of all $n$-dimensional real division algebras to decompose into four blocks. The structures of these blocks are closely related, and their relationship is made precise for a sample of full subcategories of $\mathscr{D}_{n}$.
\end{abstract}

Mathematics Subject Classification 2010: 17A35, 18B40, 54C05.

Keywords: Real division algebra, double sign, groupoid, block decomposition.

\section{Introduction}

Let $A$ be an algebra over a field $k$, i.e. a vector space over $k$ equipped with a $k$-bilinear multiplication $A \times A \rightarrow A,(x, y) \mapsto x y$. Every element $a \in A$ determines $k$-linear operators $\mathrm{L}_{a}: A \rightarrow A, x \mapsto a x$ and $\mathrm{R}_{a}: A \rightarrow A$, $x \mapsto x a$. A division algebra over $k$ is a non-zero $k$-algebra $A$ such that $\mathrm{L}_{a}$ and $\mathrm{R}_{a}$ are bijective for all $a \in A \backslash\{0\}$.

Here we concern ourselves with division algebras which are real and finite dimensional. They form a category $\mathscr{D}$ whose morphisms $\varphi: A \rightarrow B$ are non-zero linear maps satisfying $\varphi(x y)=\varphi(x) \varphi(y)$ for all $x, y \in A$. Every morphism in $\mathscr{D}$ is injective. For any positive integer $n$, the class of all $n$-dimensional objects in $\mathscr{D}$ forms a full subcategory $\mathscr{D}_{n}$ of $\mathscr{D}$. Every morphism in $\mathscr{D}_{n}$ is bijective. A famous theorem of Hopf [11], Bott and Milnor [4] and Kervaire [12] asserts that $\mathscr{D}_{n}$ is non-empty if and only if $n \in\{1,2,4,8\}$. It is well known (see e.g. [9]) that $\mathscr{D}_{1}$ consists of one isoclass only, i.e. $\mathscr{D}_{1}=[\mathbb{R}]$. In this article we derive from an elementary topological argument a decomposition of the categories $\mathscr{D}_{2}, \mathscr{D}_{4}$, and $\mathscr{D}_{8}$ into four nonempty blocks each, and we investigate the close relationship between these four blocks. Thereby we recover, unify and generalize various phenomena that previously were known in shifting disguise and for special classes of real division algebras only. 


\section{Double sign decomposition of $\mathscr{D}_{2}, \mathscr{D}_{4}$, and $\mathscr{D}_{8}$}

The sign map sign $: \mathbb{R} \backslash\{0\} \rightarrow \mathrm{C}_{2}$, $\operatorname{sign}(x)=\frac{x}{|x|}$, has its values in the cyclic group $\mathrm{C}_{2}=\{1,-1\}$. The generalized sign map

$$
s: \mathrm{GL}_{\mathbb{R}}(A) \rightarrow \mathrm{C}_{2}, s(x)=\operatorname{sign}(\operatorname{det}(x))
$$

is well defined for every finite-dimensional real vector space $A$. Being composed of group homomorphisms, it is a group homomorphism.

With any finite-dimensional real division algebra $A$ we associate the maps

$$
L: A \backslash\{0\} \rightarrow \mathrm{GL}_{\mathbb{R}}(A), a \mapsto \mathrm{L}_{a} \quad \text { and } \quad R: A \backslash\{0\} \rightarrow \mathrm{GL}_{\mathbb{R}}(A), a \mapsto \mathrm{R}_{a},
$$

whose compositions with the generalized sign map we denote by

$$
\ell: A \backslash\{0\} \rightarrow \mathrm{C}_{2}, \ell(a)=s\left(\mathrm{~L}_{a}\right) \quad \text { and } \quad r: A \backslash\{0\} \rightarrow \mathrm{C}_{2}, r(a)=s\left(\mathrm{R}_{a}\right) .
$$

Proposition 2.1. If $A \in \mathscr{D}$ has dimension greater than one, then both maps $\ell: A \backslash\{0\} \rightarrow \mathrm{C}_{2}$ and $r: A \backslash\{0\} \rightarrow \mathrm{C}_{2}$ are constant.

Proof. Equipping $A \backslash\{0\}$ with its standard Euclidean topology and $\mathrm{C}_{2}$ with the discrete topology we find that $A \backslash\{0\}$ is connected and both maps $\ell$ and $r$ are continuous, hence constant.

For any real division algebra $A$ with $1<\operatorname{dim}(A)<\infty$ we denote by $\ell(A)$ and $r(A)$ the unique values of $\ell$ and $r$ respectively. We call $\ell(A)$ the left sign of $A, r(A)$ the right sign of $A$, and $p(A)=(\ell(A), r(A))$ the sign pair of $A$.

Proposition 2.2. If $A, B \in \mathscr{D}$ have dimension greater than one and are isomorphic, then $p(A)=p(B)$.

Proof. Let $\varphi: A \rightarrow B$ be an isomorphism in $\mathscr{D}$. Choose $a \in A \backslash\{0\}$ and set $b=\varphi(a)$. Then $\mathrm{L}_{b}=\varphi \mathrm{L}_{a} \varphi^{-1}$ implies $\operatorname{det}\left(\mathrm{L}_{b}\right)=\operatorname{det}\left(\mathrm{L}_{a}\right)$, whence $\ell(B)=\ell(b)=\operatorname{sign}\left(\operatorname{det}\left(\mathrm{L}_{b}\right)\right)=\operatorname{sign}\left(\operatorname{det}\left(\mathrm{L}_{a}\right)\right)=\ell(a)=\ell(A)$. Likewise, $\mathrm{R}_{b}=\varphi \mathrm{R}_{a} \varphi^{-1}$ implies $r(B)=r(A)$.

Together the above two propositions assert that the sign pair map

$$
p: \mathscr{D} \backslash[\mathbb{R}] \rightarrow \mathrm{C}_{2} \times \mathrm{C}_{2}, p(A)=(\ell(A), r(A))
$$

is well defined, and constant on all isoclasses. For each $n \in\{2,4,8\}$ we denote its restriction to $\mathscr{D}_{n}$ by $p_{n}: \mathscr{D}_{n} \rightarrow \mathrm{C}_{2} \times \mathrm{C}_{2}$. For each $(\alpha, \beta) \in \mathrm{C}_{2} \times \mathrm{C}_{2}$, the fibre $p_{n}^{-1}(\alpha, \beta)$ forms a full subcategory $\mathscr{D}_{n}^{\alpha, \beta}$ of $\mathscr{D}_{n}$. Usually we prefer the more intuitive notation

$$
\mathscr{D}_{n}^{++}=\mathscr{D}_{n}^{1,1}, \mathscr{D}_{n}^{+-}=\mathscr{D}_{n}^{1,-1}, \mathscr{D}_{n}^{-+}=\mathscr{D}_{n}^{-1,1}, \quad \text { and } \quad \mathscr{D}_{n}^{--}=\mathscr{D}_{n}^{-1,-1} .
$$


Proposition 2.3. For each $n \in\{2,4,8\}$, the category $\mathscr{D}_{n}$ decomposes in accordance with

$$
\mathscr{D}_{n}=\coprod_{(\alpha, \beta) \in \mathrm{C}_{2} \times \mathrm{C}_{2}} \mathscr{D}_{n}^{\alpha, \beta}
$$

Proof. The object class of $\mathscr{D}_{n}$ is the disjoint union of the fibres of $p_{n}$, i.e. the disjoint union of the object classes of $\mathscr{D}_{n}^{\alpha, \beta}$, where $(\alpha, \beta) \in \mathrm{C}_{2} \times \mathrm{C}_{2}$.

Every morphism $\varphi: A \rightarrow B$ in $\mathscr{D}_{n}$ is an isomorphism, and $A \in \mathscr{D}_{n}^{\alpha, \beta}$ and $B \in \mathscr{D}_{n}^{\gamma, \delta}$ for $(\alpha, \beta)=p_{n}(A)$ and $(\gamma, \delta)=p_{n}(B)$. Proposition 2.2 implies that $(\alpha, \beta)=(\gamma, \delta)$, whence $\varphi$ is a morphism in $\mathscr{D}_{n}^{\alpha, \beta}$.

\section{Relationships between the blocks $\mathscr{D}_{n}^{\alpha, \beta}$ of $\mathscr{D}_{n}$}

The purpose of this section is to exhibit two types of relationships between the four blocks $\mathscr{D}_{n}^{\alpha, \beta}$ of $\mathscr{D}_{n}$. The first of these is provided by opposition and described in the following proposition, the proof of which is straightforward.

Proposition 3.1. (i) The passage from an algebra $A \in \mathscr{D}$ to its opposite algebra $A^{\mathrm{op}}$ yields an endofunctor $\mathscr{O}: \mathscr{D} \rightarrow \mathscr{D}$, defined on morphisms $\varphi: A \rightarrow B$ by $\mathscr{O}(\varphi)=\varphi$.

(ii) The endofunctor $\mathscr{O}$ is a self-inverse automorphism of $\mathscr{D}$. It induces for all $n \in\{2,4,8\}$ and $(\alpha, \beta) \in \mathrm{C}_{2} \times \mathrm{C}_{2}$ an isomorphism of blocks

$$
\mathscr{O}_{n}^{\alpha, \beta}: \mathscr{D}_{n}^{\alpha, \beta} \rightarrow \mathscr{D}_{n}^{\beta, \alpha},
$$

with inverse isomorphism $\mathscr{O}_{n}^{\beta, \alpha}$.

The second type of relationship lies deeper. For any fixed $n \in\{2,4,8\}$ and $m \in \mathbb{N}$ odd with $m<n$ we introduce the category $\mathscr{D}_{m n}$, whose objects are triples $(A, U, V)$ formed by an algebra $A \in \mathscr{D}_{n}$ and supplementary subspaces $U, V \subset A$ of dimensions $m$ and $n-m$ respectively. A morphism $\varphi:(A, U, V) \rightarrow\left(A^{\prime}, U^{\prime}, V^{\prime}\right)$ in $\mathscr{D}_{m n}$ is a morphism $\varphi: A \rightarrow A^{\prime}$ in $\mathscr{D}_{n}$ satisfying $\varphi(U)=U^{\prime}$ and $\varphi(V)=V^{\prime}$.

The relevance of $\mathscr{D}_{m n}$ to $\mathscr{D}_{n}$ is explained by the forgetful functor $\mathscr{F}: \mathscr{D}_{m n} \rightarrow \mathscr{D}_{n}$, defined on objects by $\mathscr{F}(A, U, V)=A$ and on morphisms by $\mathscr{F}(\varphi)=\varphi$. This functor $\mathscr{F}$ is faithful and dense. For all $(\alpha, \beta) \in \mathrm{C}_{2} \times \mathrm{C}_{2}$ we denote by $\mathscr{D}_{m n}^{\alpha, \beta}$ the full subcategory of $\mathscr{D}_{m n}$ that is formed by all objects $(A, U, V) \in \mathscr{D}_{m n}$ with $A \in \mathscr{D}_{n}^{\alpha, \beta}$. Now $\mathscr{F}$ induces forgetful functors $\mathscr{F}^{\alpha, \beta}: \mathscr{D}_{m n}^{\alpha, \beta} \rightarrow \mathscr{D}_{n}^{\alpha, \beta}$, which retain the property of being faithful and dense. For the remainder of this section we shift our focus from $\mathscr{D}_{n}^{\alpha, \beta}$ to $\mathscr{D}_{m n}^{\alpha, \beta}$, ultimately proving that, for fixed $(m, n)$ and varying $(\alpha, \beta)$, all categories $\mathscr{D}_{m n}^{\alpha, \beta}$ are isomorphic (Corollary 3.5).

To begin with, recall that the isotope of a $k$-algebra $A$ with respect to $(\sigma, \tau) \in \mathrm{GL}_{k}(A) \times \mathrm{GL}_{k}(A)$ is the $k$-algebra $A_{\sigma, \tau}$ with underlying vector space $A$, and multiplication $x \circ y=\sigma(x) \tau(y)$. Thus $\left(A_{\sigma, \tau}\right)_{\sigma^{\prime}, \tau^{\prime}}=A_{\sigma \sigma^{\prime}, \tau \tau^{\prime}}$ 
holds for all $(\sigma, \tau),\left(\sigma^{\prime}, \tau^{\prime}\right) \in \mathrm{GL}_{k}(A) \times \mathrm{GL}_{k}(A)$. Also, every element $a \in$ $A \backslash\{0\}$ determines $k$-linear operators $\mathrm{L}_{a}^{\circ}: A_{\sigma, \tau} \rightarrow A_{\sigma, \tau}, \mathrm{L}_{a}^{\circ}(x)=a \circ x$ and $\mathrm{R}_{a}^{\circ}: A_{\sigma, \tau} \rightarrow A_{\sigma, \tau}, \mathrm{R}_{a}^{\circ}(x)=x \circ a$ which, by definition of isotopy, satisfy the identities $\mathrm{L}_{a}^{\circ}=\mathrm{L}_{\sigma(a)} \tau$ and $\mathrm{R}_{a}^{\circ}=\mathrm{R}_{\tau(a)} \sigma$. It follows that $A_{\sigma, \tau}$ is a division algebra if so is $A$.

Lemma 3.2. Let $A \in \mathscr{D}_{n}$ for some $n \in\{2,4,8\}$, and let $(\sigma, \tau) \in\left(\mathrm{GL}_{\mathbb{R}}(A)\right)^{2}$. If $p_{n}(A)=(\alpha, \beta)$, then $p_{n}\left(A_{\sigma, \tau}\right)=(\alpha, \beta)(s(\tau), s(\sigma))$.

Proof. Choosing any element $a \in A \backslash\{0\}$, we have that

$$
\ell\left(A_{\sigma, \tau}\right)=s\left(\mathrm{~L}_{a}^{\circ}\right)=s\left(\mathrm{~L}_{\sigma(a)} \tau\right)=s\left(\mathrm{~L}_{\sigma(a)}\right) s(\tau)=\ell(A) s(\tau)=\alpha s(\tau)
$$

and likewise

$$
r\left(A_{\sigma, \tau}\right)=s\left(\mathrm{R}_{a}^{\circ}\right)=s\left(\mathrm{R}_{\tau(a)} \sigma\right)=s\left(\mathrm{R}_{\tau(a)}\right) s(\sigma)=r(A) s(\sigma)=\beta s(\sigma) .
$$

Every object $X=(A, U, V) \in \mathscr{D}_{m n}$ determines an invertible linear operator $\kappa=\kappa_{X} \in \mathrm{GL}_{\mathbb{R}}(A)$, defined by $\kappa(u+v)=u-v$, where $u \in U$ and $v \in V$.

Lemma 3.3. If $\varphi: X \rightarrow X^{\prime}$ is a morphism in $\mathscr{D}_{m n}$, then $\varphi \kappa=\kappa^{\prime} \varphi$.

Proof. Let $X=(A, U, V)$ and $X^{\prime}=\left(A^{\prime}, U^{\prime}, V^{\prime}\right)$. Every $x \in A$ has a unique decomposition $x=u+v$, with $u \in U$ and $v \in V$. Since $\varphi(u) \in U^{\prime}$ and $\varphi(v) \in V^{\prime}$, we obtain

$$
\begin{aligned}
\varphi \kappa(x) & =\varphi \kappa(u+v)=\varphi(u-v)=\varphi(u)-\varphi(v) \\
& =\kappa^{\prime}(\varphi(u)+\varphi(v))=\kappa^{\prime} \varphi(u+v)=\kappa^{\prime} \varphi(x) .
\end{aligned}
$$

Theorem 3.4. (i) For each $(i, j) \in\{0,1\}^{2}$, the passage from $X=(A, U, V)$ in $\mathscr{D}_{m n}$ to $\mathscr{I}_{i j}(X)=\left(A_{\kappa^{i}, \kappa^{j}}, U, V\right)$ yields an endofunctor $\mathscr{I}_{i j}: \mathscr{D}_{m n} \rightarrow \mathscr{D}_{m n}$, defined on morphisms $\varphi: X \rightarrow X^{\prime}$ by $\mathscr{I}_{i j}(\varphi)=\varphi$.

(ii) The set of endofunctors $\mathscr{I}=\left\{\mathscr{I}_{i j} \mid(i, j) \in\{0,1\}^{2}\right\}$ forms a group under composition, and the map

$$
\mathrm{C}_{2} \times \mathrm{C}_{2} \rightarrow \mathscr{I},\left((-1)^{i},(-1)^{j}\right) \mapsto \mathscr{I}_{i j}
$$

is a group isomorphism.

(iii) Each of the four automorphisms $\mathscr{I}_{i j}: \mathscr{D}_{m n} \rightarrow \mathscr{D}_{m n}$ induces isomorphisms of blocks

$$
\mathscr{I}_{i j}^{\alpha, \beta}: \mathscr{D}_{m n}^{\alpha, \beta} \rightarrow \mathscr{D}_{m n}^{(-1)^{j} \alpha,(-1)^{i} \beta},
$$

for all $(\alpha, \beta) \in \mathrm{C}_{2} \times \mathrm{C}_{2}$. 
Proof. (i) If $X \in \mathscr{D}_{m n}$, then $\mathscr{I}_{i j}(X) \in \mathscr{D}_{m n}$. If $\varphi: X \rightarrow X^{\prime}$ is a morphism in $\mathscr{D}_{m n}$, then also $\varphi: \mathscr{I}_{i j}(X) \rightarrow \mathscr{I}_{i j}\left(X^{\prime}\right)$ is a morphism in $\mathscr{D}_{m n}$, by Lemma 3.3 .

(ii) Denoting the identity functor on $\mathscr{D}_{m n}$ by Id, we have the equalities

$$
\mathrm{Id}=\mathscr{I}_{00}=\mathscr{I}_{10}^{2}=\mathscr{I}_{01}^{2} \quad \text { and } \quad \mathscr{I}_{10} \mathscr{I}_{01}=\mathscr{I}_{11}=\mathscr{I}_{01} \mathscr{I}_{10}
$$

while $\mathscr{I}_{10} \neq \mathrm{Id}, \mathscr{I}_{01} \neq \mathrm{Id}$, and $\mathscr{I}_{11} \neq \mathrm{Id}$.

(iii) Given $(m, n),(i, j)$ and $(\alpha, \beta)$ as in the statement, let $X=(A, U, V)$ be an object in $\mathscr{D}_{m n}^{\alpha, \beta}$. Observing that

$$
s(\kappa)=\operatorname{sign}(\operatorname{det}(\kappa))=\operatorname{sign}\left((-1)^{n-m}\right)=\operatorname{sign}(-1)=-1,
$$

we obtain with Lemma 3.2 that

$$
p_{n}\left(A_{\kappa^{i}, \kappa^{j}}\right)=(\alpha, \beta)\left(s\left(\kappa^{j}\right), s\left(\kappa^{i}\right)\right)=(\alpha, \beta)\left((-1)^{j},(-1)^{i}\right),
$$

which means that $\mathscr{I}_{i j}(X) \in \mathscr{D}_{m n}^{(-1)^{j} \alpha,(-1)^{i} \beta}$. Hence the automorphism $\mathscr{I}_{i j}: \mathscr{D}_{m n} \rightarrow \mathscr{D}_{m n}$ induces a functor

$$
\mathscr{I}_{i j}^{\alpha, \beta}: \mathscr{D}_{m n}^{\alpha, \beta} \rightarrow \mathscr{D}_{m n}^{(-1)^{j} \alpha,(-1)^{i} \beta},
$$

which in fact is an isomorphism with inverse functor

$$
\mathscr{I}_{i j}^{(-1)^{j} \alpha,(-1)^{i} \beta}: \mathscr{D}_{m n}^{(-1)^{j} \alpha,(-1)^{i} \beta} \rightarrow \mathscr{D}_{m n}^{\alpha, \beta} .
$$

Corollary 3.5. For each $n \in\{2,4,8\}$ and $m \in \mathbb{N}$ odd with $m<n$, the category $\mathscr{D}_{m n}$ decomposes in accordance with

$$
\mathscr{D}_{m n}=\coprod_{(\alpha, \beta) \in \mathrm{C}_{2} \times \mathrm{C}_{2}} \mathscr{D}_{m n}^{\alpha, \beta},
$$

and all its four blocks $\mathscr{D}_{m n}^{\alpha, \beta}$ are isomorphic. More precisely, if $(\alpha, \beta),(\gamma, \delta) \in$ $\mathrm{C}_{2} \times \mathrm{C}_{2}$, then $\mathscr{I}_{i j}^{\alpha, \beta}: \mathscr{D}_{m n}^{\alpha, \beta} \rightarrow \mathscr{D}_{m n}^{\gamma, \delta}$ is an isomorphism for the unique pair $(i, j) \in\{0,1\}^{2}$ satisfying $\left((-1)^{i},(-1)^{j}\right)=(\delta \beta, \gamma \alpha)$.

Proof. The stated decomposition of $\mathscr{D}_{m n}$ is an immediate consequence of Proposition 2.3. Moreover, $\mathscr{I}_{i j}^{\alpha, \beta}: \mathscr{D}_{m n}^{\alpha, \beta} \rightarrow \mathscr{D}_{m n}^{(-1)^{j} \alpha,(-1)^{i} \beta}$ is an isomorphism of categories by Theorem 3.4(iii), and $\mathscr{D}_{m n}^{(-1)^{j} \alpha,(-1)^{i} \beta}=\mathscr{D}_{m n}^{\gamma, \delta}$ provided that $\left((-1)^{i},(-1)^{j}\right)=\left(\delta \beta^{-1}, \gamma \alpha^{-1}\right)$.

Composing the forgetful functors $\mathscr{F}^{\alpha, \beta}$ with the isomorphisms $\mathscr{I}_{i j}^{\alpha, \beta}$, we arrive at the announced second type of relationship between the blocks $\mathscr{D}_{n}^{\alpha, \beta}$ of $\mathscr{D}_{n}$. 
Corollary 3.6. Let $n \in\{2,4,8\}$ and $m \in \mathbb{N}$ odd with $m<n$. Given $(\alpha, \beta),(\gamma, \delta) \in \mathrm{C}_{2} \times \mathrm{C}_{2}$, let $(i, j) \in\{0,1\}^{2}$ be the unique pair satisfying $\left((-1)^{i},(-1)^{j}\right)=(\delta \beta, \gamma \alpha)$. Then the blocks $\mathscr{D}_{n}^{\alpha, \beta}$ and $\mathscr{D}_{n}^{\gamma, \delta}$ of $\mathscr{D}_{n}$ are related by the diagram

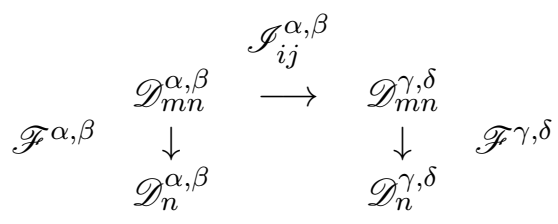

where the isotopy functor $\mathscr{I}_{i j}^{\alpha, \beta}$ is an isomorphism of categories, while the forgetful functors $\mathscr{F}^{\alpha, \beta}$ and $\mathscr{F}^{\gamma, \delta}$ are faithful and dense.

For suitable full subcategories $\mathscr{C}_{n} \subset \mathscr{D}_{n}$, the diagram of Corollary 3.6 induces isomorphisms of blocks $\mathscr{C}_{n}^{\alpha, \beta}$ and $\mathscr{C}_{n}^{\gamma, \delta}$. This is discussed in the next section (Lemma 4.2).

\section{Blocks of full subcategories of $\mathscr{D}_{n}$}

\subsection{Generalities}

Let $\mathscr{C} \subset \mathscr{D}$ be a full subcategory. For all $n \in\{2,4,8\}$ and $(\alpha, \beta) \in \mathrm{C}_{2} \times \mathrm{C}_{2}$ we denote by $\mathscr{C}_{n}$ and $\mathscr{C}_{n}^{\alpha, \beta}$ the full subcategories of $\mathscr{C}$ whose object classes are $\mathscr{C} \cap \mathscr{D}_{n}$ and $\mathscr{C} \cap \mathscr{D}_{n}^{\alpha, \beta}$ respectively. Now Proposition 2.3 implies that

$$
\mathscr{C}_{n}=\coprod_{(\alpha, \beta) \in \mathrm{C}_{2} \times \mathrm{C}_{2}} \mathscr{C}_{n}^{\alpha, \beta} .
$$

In case $\mathscr{C}$ is one of the full subcategories $\mathscr{D}^{c}, \mathscr{D}^{\ell}, \mathscr{D}^{r}$, and $\mathscr{D}^{1}$ of $\mathscr{D}$ which are formed by all algebras $A \in \mathscr{D}$ having non-zero centre, a left unity, a right unity, and a unity respectively, Proposition 2.1 implies that two or three of the blocks $\mathscr{C}_{n}^{\alpha, \beta}$ are empty, and accordingly the above decomposition of $\mathscr{C}_{n}$ takes on the following special forms.

Corollary 4.1. For all $n \in\{2,4,8\}$, the categories $\mathscr{D}_{n}^{c}, \mathscr{D}_{n}^{\ell}, \mathscr{D}_{n}^{r}$, and $\mathscr{D}_{n}^{1}$ decompose according to

(i) $\mathscr{D}_{n}^{c}=\mathscr{D}_{n}^{c++} \amalg \mathscr{D}_{n}^{c--}$,

(ii) $\mathscr{D}_{n}^{\ell}=\mathscr{D}_{n}^{\ell++} \amalg \mathscr{D}_{n}^{\ell+-}$,

(iii) $\mathscr{D}_{n}^{r}=\mathscr{D}_{n}^{r++} \amalg \mathscr{D}_{n}^{r-+}$, and

(iv) $\mathscr{D}_{n}^{1}=\mathscr{D}_{n}^{1++}$.

Given any full subcategory $\mathscr{C}_{n} \subset \mathscr{D}_{n}$, when are two blocks $\mathscr{C}_{n}^{\alpha, \beta}$ and $\mathscr{C}_{n}^{\gamma, \delta}$ of $\mathscr{C}_{n}$ equivalent? A sufficient criterion is presented in the following lemma, and applied to $e$-quadratic real division algebras in the next subsection. 
Lemma 4.2. Let $n \in\{2,4,8\}$ and $\mathscr{C}_{n} \subset \mathscr{D}_{n}$ be any full subcategory. If there exist $m \in \mathbb{N}$ odd with $m<n$ and a functor $\mathscr{G}: \mathscr{C}_{n} \rightarrow \mathscr{D}_{m n}$ such that $\mathscr{F} \mathscr{G}=$ Id and $\mathscr{I}_{i j}\left(\mathscr{G}\left(\mathscr{C}_{n}\right)\right) \subset \mathscr{G}\left(\mathscr{C}_{n}\right)$ for some $(i, j) \in\{0,1\}^{2}$, then the blocks $\mathscr{C}_{n}^{\alpha, \beta}$ and $\mathscr{C}_{n}^{(-1)^{j} \alpha,(-1)^{i} \beta}$ are isomorphic for all $(\alpha, \beta) \in \mathrm{C}_{2} \times \mathrm{C}_{2}$.

Proof. By hypothesis there is a functor $\mathscr{G}: \mathscr{C}_{n} \rightarrow \mathscr{D}_{m n}$ satisfying $\mathscr{F} \mathscr{G}=\mathrm{Id}$. Accordingly $\mathscr{G}(A)=\left(A, U_{A}, V_{A}\right)$ for all objects $A \in \mathscr{C}_{n}$, and $\mathscr{G}(\varphi)=\varphi$ for all morphisms $\varphi: A \rightarrow B$ in $\mathscr{C}_{n}$. The full subcategory $\mathscr{G}\left(\mathscr{C}_{n}\right) \subset \mathscr{D}_{m n}$ is thus isomorphic to $\mathscr{C}_{n}$, with mutually inverse isomorphisms

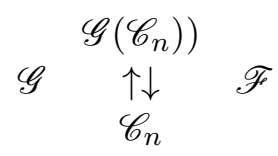

induced by the given functor $\mathscr{G}: \mathscr{C}_{n} \rightarrow \mathscr{D}_{m n}$ and the forgetful functor $\mathscr{F}: \mathscr{D}_{m n} \rightarrow \mathscr{D}_{n}$. The hypothesis $\mathscr{I}_{i j}\left(\mathscr{G}\left(\mathscr{C}_{n}\right)\right) \subset \mathscr{G}\left(\mathscr{C}_{n}\right)$ implies together with Theorem 3.4(ii) that the automorphism $\mathscr{I}_{i j}: \mathscr{D}_{m n} \rightarrow \mathscr{D}_{m n}$ induces an automorphism $\mathscr{I}_{i j}: \mathscr{G}\left(\mathscr{C}_{n}\right) \rightarrow \mathscr{G}\left(\mathscr{C}_{n}\right)$. This establishes the sequence of isomorphisms

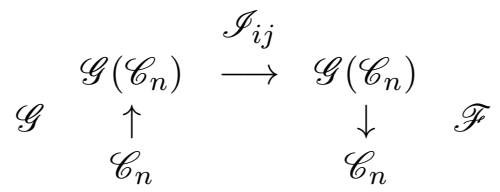

which in view of Theorem 3.4(iii) induces a sequence of isomorphisms

$$
\begin{array}{ccccc} 
& & \mathscr{I}_{i j}^{\alpha, \beta} & & \\
& \mathscr{G}\left(\mathscr{C}_{n}^{\alpha, \beta}\right) & \stackrel{ }{\longrightarrow} & \mathscr{G}\left(\mathscr{C}_{n}^{\gamma, \delta}\right) & \\
\mathscr{G}^{\alpha, \beta} & \uparrow & & \downarrow & \\
& \uparrow & & \mathscr{F}^{\gamma, \delta} \\
& \mathscr{C}_{n}^{\alpha, \beta} & & \mathscr{C}_{n}^{\gamma, \delta} & \\
& & &
\end{array}
$$

for every $(\alpha, \beta) \in \mathrm{C}_{2} \times \mathrm{C}_{2}$, where $(\gamma, \delta)=\left((-1)^{j} \alpha,(-1)^{i} \beta\right)$.

\subsection{Blocks of $e$-quadratic real division algebras}

Following Cuenca Mira [5], an algebra $A$ over a field $k$ is called $e$-quadratic if it contains a non-zero central idempotent $e$ such that $x^{2} \in \operatorname{span}_{k}\{e, e x\}$ for all $x \in A$. For any $k$-algebra $A$ with non-zero central idempotent $e$ we define the subset $\operatorname{Im}_{e}(A) \subset A$ by $\operatorname{Im}_{e}(A)=\left\{v \in A \backslash k e \mid v^{2} \in k e\right\} \cup\{0\}$. Then "Frobenius's trick" [10, p. 61] leads to a proof of the subsequent Lemma 4.3 (cf. also [7, Lemma 1], [13, p. 227]).

Lemma 4.3. If $A$ is an e-quadratic $k$-algebra with $\operatorname{char}(k) \neq 2$ and $\mathrm{L}_{e}$ is injective, then $\operatorname{Im}_{e}(A) \subset A$ is a k-linear subspace and $A=k e \oplus \operatorname{Im}_{e}(A)$.

Lemma 4.4. If $A$ is an e-quadratic real division algebra and $\operatorname{dim}(A)>2$, then $e$ is unique. In particular, the decomposition $A=\mathbb{R e} \oplus \operatorname{Im}_{e}(A)$ is uniquely determined by $A$. 
Proof. Let $f \in A$ be any non-zero central idempotent such that $x^{2} \in$ $\operatorname{span}_{\mathbb{R}}\{f, f x\}$ for all $x \in A$. Then Lemma 4.3 combined with $\operatorname{dim}(A)>2 \mathrm{im}$ plies that $\operatorname{Im}_{e}(A) \cap \operatorname{Im}_{f}(A)$ contains a non-zero element $v$, and $v^{2} \in \mathbb{R} e \cap \mathbb{R} f$ together with $v^{2} \neq 0$ implies $e=f$.

Consider now the full subcategory $\mathscr{E} \subset \mathscr{D}$ that is formed by all $e$-quadratic algebras in $\mathscr{D}$. Let $n \in\{4,8\}$ and $A, B \in \mathscr{E}_{n}$. By Lemma 4.4, $A$ and $B$ have unique decompositions $A=\mathbb{R} e \oplus \operatorname{Im}_{e}(A)$ and $B=\mathbb{R} f \oplus \operatorname{Im}_{f}(B)$ respectively. Moreover, every morphism $\varphi: A \rightarrow B$ in $\mathscr{E}_{n}$ satisfies $\varphi(e)=f$ and $\varphi\left(\operatorname{Im}_{e}(A)\right)=\operatorname{Im}_{f}(B)$. So $\varphi:\left(A, \mathbb{R} e, \operatorname{Im}_{e}(A)\right) \rightarrow\left(B, \mathbb{R} f, \operatorname{Im}_{f}(B)\right)$ is a morphism in $\mathscr{D}_{1 n}$. This reveals a functor $\mathscr{G}: \mathscr{E}_{n} \rightarrow \mathscr{D}_{1 n}$, defined on objects by $\mathscr{G}(A)=\left(A, \mathbb{R} e, \operatorname{Im}_{e}(A)\right)$ and on morphisms by $\mathscr{G}(\varphi)=\varphi$.

Proposition 4.5. For each $n \in\{4,8\}$, the functor $\mathscr{G}: \mathscr{E}_{n} \rightarrow \mathscr{D}_{1 n}$ satisfies $\mathscr{F} \mathscr{G}=\operatorname{Id}$ and $\mathscr{I}_{11}\left(\mathscr{G}\left(\mathscr{E}_{n}\right)\right) \subset \mathscr{G}\left(\mathscr{E}_{n}\right)$.

Proof. The identity $\mathscr{F} \mathscr{G}=$ Id holds by definition of $\mathscr{G}$. To prove the asserted inclusion, let $A \in \mathscr{E}_{n}$. Then

$$
\mathscr{I}_{11}(\mathscr{G}(A))=\mathscr{I}_{11}\left(A, \mathbb{R} e, \operatorname{Im}_{e}(A)\right)=\left(A_{\kappa, \kappa}, \mathbb{R} e, \operatorname{Im}_{e}(A)\right),
$$

where $\kappa(\alpha e+v)=\alpha e-v$ for all $\alpha \in \mathbb{R}$ and $v \in \operatorname{Im}_{e}(A)$. Now $e \in A_{\kappa, \kappa}$ is a non-zero central idempotent such that $x \circ x=\kappa(x) \kappa(x) \in \operatorname{span}_{\mathbb{R}}\{e, e \kappa(x)\}=$ $\operatorname{span}_{\mathbb{R}}\{e, e \circ x\}$ for all $x \in A_{\kappa, \kappa}$, and $v \circ v=(-v)(-v)=v^{2} \in \mathbb{R} e$ for all $v \in \operatorname{Im}_{e}(A)$. Hence $A_{\kappa, \kappa} \in \mathscr{E}_{n}$, and $\operatorname{Im}_{e}(A)=\operatorname{Im}_{e}\left(A_{\kappa, \kappa}\right)$. It follows that $\mathscr{I}_{11}(\mathscr{G}(A))=\left(A_{\kappa, \kappa}, \mathbb{R} e, \operatorname{Im}_{e}\left(A_{\kappa, \kappa}\right)\right)=\mathscr{G}\left(A_{\kappa, \kappa}\right)$.

Corollary 4.6. For each $n \in\{4,8\}$, the category $\mathscr{E}_{n}$ of all $n$-dimensional e-quadratic real division algebras decomposes in accordance with

$$
\mathscr{E}_{n}=\mathscr{E}_{n}^{++} \amalg \mathscr{E}_{n}^{--},
$$

and its blocks $\mathscr{E}_{n}^{++}$and $\mathscr{E}_{n}^{--}$are isomorphic.

Proof. Since $\mathscr{E}_{n} \subset \mathscr{D}_{n}^{c}$, the asserted block decomposition of $\mathscr{E}_{n}$ follows from Corollary 4.1(i). Proposition 4.5 states that Lemma 4.2 can be applied to the full subcategory $\mathscr{E}_{n} \subset \mathscr{D}_{n}, m=1$ and $(i, j)=(1,1)$, which yields the isomorphism of blocks $\mathscr{E}_{n}^{++}$and $\mathscr{E}_{n}^{--}$.

\subsection{Blocks of isotopes of the quaternion algebra}

We denote by $\mathcal{Q}$ the full subcategory of $\mathscr{D}_{4}$ consisting of all isotopes of Hamilton's quaternion algebra $\mathbb{H}$, i.e., all algebras of the form $\mathbb{H}_{\sigma, \tau}$ for some $\sigma, \tau \in \mathrm{GL}_{\mathbb{R}}(\mathbb{H})$. Moreover, $\mathscr{P}$ is the full subcategory $\mathscr{P}=\left\{\mathbb{H}_{\sigma, \tau} \mid \sigma, \tau \in\right.$ $\mathrm{O}(\mathbb{H})\} \subset \mathcal{Q}$.

An absolute valued algebra is a real algebra $A$ equipped with a norm $\|\cdot\|: A \rightarrow \mathbb{R}$ satisfying $\|x y\|=\|x\|\|y\|$ for all $x, y \in A$. Due to Albert [2], 
every finite-dimensional absolute valued algebra is isomorphic to an isotope $A_{\sigma, \tau}$, where $A$ is one of the four classical real division algebras $\mathbb{R}, \mathbb{C}, \mathbb{H}$ and $\mathbb{O}$, and $\sigma, \tau \in \mathrm{O}(A)$. Taking $\mathscr{A}$ to be the category of all finite-dimensional absolute valued algebras, it follows that $\mathscr{P}$ is a dense and full subcategory of $\mathscr{A}_{4}$.

For any ring $R$, the multiplicative group of invertible elements in $R$ is denoted by $R^{*}$. Every left group action $G \times M \rightarrow M$ gives rise to a groupoid ${ }_{G} M$, with object set $O b\left({ }_{G} M\right)=M$ and morphism sets ${ }_{G} M(x, y)=$ $\{(g, x, y) \mid g x=y\}$ for all $x, y \in M$. In particular, the action of $\mathbb{H}^{*} / \mathbb{R}^{*}$ on the set $\left(\mathbb{H}^{*} / \mathbb{R}^{*}\right)^{2} \times \mathscr{S}_{\mathbb{H}}^{2}$ given by

$$
[s] \cdot(([a],[b]),(C, D))=\left(\left(\left[K_{s}(a)\right],\left[K_{s}(b)\right]\right),\left(K_{s} C K_{s}^{-1}, K_{s} D K_{s}^{-1}\right)\right)
$$

gives rise to a groupoid $\mathscr{Z}={ }_{\mathbb{H}^{*}} / \mathbb{R}^{*}\left(\left(\mathbb{H}^{*} / \mathbb{R}^{*}\right)^{2} \times \mathscr{S}_{\mathbb{H}}^{2}\right)$. Here $\mathscr{S}_{\mathbb{H}}$ denotes the set of all linear endomorphisms of $\mathbb{H}$ that are positive definite symmetric and have determinant 1 , and $K_{s}=\mathrm{L}_{s} \mathrm{R}_{s^{-1}}$.

Let $\kappa$ be the natural involution on $\mathbb{H}$, i.e., $\kappa=\kappa_{X}$ for $X=\left(\mathbb{H}, \mathbb{R} 1, \operatorname{Im}_{1}(\mathbb{H})\right)$ $\in \mathscr{D}_{14}\left(\mathbb{H}\right.$ is quadratic, so by Lemma $4.3, \operatorname{Im}_{1}(\mathbb{H}) \subset \mathbb{H}$ is a subspace complementary to $\mathbb{R} 1$ ). Choose a set $\mathcal{H}$ of coset representatives for $\mathbb{H}^{*} / \mathbb{R}^{*}$, such that every element of $\mathcal{H}$ has norm 1 . For $(\alpha, \beta) \in \mathrm{C}_{2} \times \mathrm{C}_{2}$, let $\mathscr{H}_{\alpha, \beta}: \mathscr{Z} \rightarrow \mathcal{Q}^{\alpha, \beta}$ be the functor defined by $\mathscr{H}_{\alpha, \beta}([s])=K_{s}$ for morphisms, and for objects $\mathscr{H}_{\alpha, \beta}(([a],[b]),(C, D))=\mathbb{H}_{\sigma, \tau}$, where $a, b \in \mathcal{H}$ and

$$
\begin{array}{ll}
(\sigma, \tau)=\left(\mathrm{L}_{a} C, \mathrm{R}_{b} D\right) & \text { if } \quad(\alpha, \beta)=(1,1), \\
(\sigma, \tau)=\left(\mathrm{R}_{a} C \kappa, \mathrm{R}_{b} D\right) & \text { if } \quad(\alpha, \beta)=(1,-1), \\
(\sigma, \tau)=\left(\mathrm{L}_{a} C, \mathrm{~L}_{b} D \kappa\right) & \text { if } \quad(\alpha, \beta)=(-1,1), \\
(\sigma, \tau)=\left(\mathrm{L}_{a} C \kappa, \mathrm{R}_{b} D \kappa\right) & \text { if } \quad(\alpha, \beta)=(-1,-1) .
\end{array}
$$

We have the following result (see [6, Propositions 11, 12]):

Proposition 4.7. For each $(\alpha, \beta) \in \mathrm{C}_{2} \times \mathrm{C}_{2}$, the functor $\mathscr{H}_{\alpha, \beta}: \mathscr{Z} \rightarrow \mathcal{Q}^{\alpha, \beta}$ is an equivalence of categories. Thus the four blocks $\mathcal{Q}^{++}, \mathcal{Q}^{+-} \mathcal{Q}^{-+}$and $\mathcal{Q}^{--}$are equivalent to each other.

Let $\mathscr{Y} \subset \mathscr{Z}$ be the full subcategory defined by $\mathscr{Y}={ }_{\mathbb{H}^{*}} / \mathbb{R}^{*}\left(\left(\mathbb{H}^{*} / \mathbb{R}^{*}\right)^{2} \times\{\mathbb{I}\}^{2}\right)$.

Corollary 4.8. For each $(\alpha, \beta) \in \mathrm{C}_{2} \times \mathrm{C}_{2}$, the functor $\mathscr{H}_{\alpha, \beta}$ induces an equivalence $\mathscr{Y} \rightarrow \mathscr{P}^{\alpha, \beta}$. Hence the four blocks $\mathscr{A}_{4}^{++}, \mathscr{A}_{4}^{+-}, \mathscr{A}_{4}^{-+}$and $\mathscr{A}_{4}^{--}$ are equivalent to each other.

Proof. For the first statement, it suffices to observe that $\mathscr{Y}=\mathscr{H}_{\alpha, \beta}^{-1}\left(\mathscr{P}^{\alpha, \beta}\right)$ for all $(\alpha, \beta) \in \mathrm{C}_{2} \times \mathrm{C}_{2}$. Since $\mathscr{P}$ is dense in $\mathscr{A}_{4}, \mathscr{P}^{\alpha, \beta}$ is dense in $\mathscr{A}_{4}^{\alpha, \beta}$, and

$$
\mathscr{A}_{4}^{\alpha, \beta} \simeq \mathscr{P}^{\alpha, \beta} \simeq \mathscr{Y} \simeq \mathscr{P}^{\alpha^{\prime}, \beta^{\prime}} \simeq \mathscr{A}_{4}^{\alpha^{\prime}, \beta^{\prime}}
$$

for all $(\alpha, \beta),\left(\alpha^{\prime}, \beta^{\prime}\right) \in \mathrm{C}_{2} \times \mathrm{C}_{2}$. 


\subsection{Blocks of 2-dimensional real division algebras}

Let $A$ be a division algebra over a field $k$. For each $a \in A \backslash\{0\}$, the isotope $A_{\mathrm{R}_{a}^{-1}, \mathrm{~L}_{a}^{-1}}$ has unity $a^{2}[1$, Theorem 7$]$. In case $\operatorname{dim}(A)=2$ it follows that $A_{\mathrm{R}_{a}^{-1}, \mathrm{~L}_{a}^{-1}}$ is quadratic, which for $k=\mathbb{R}$ means that $A_{\mathrm{R}_{a}^{-1}, \mathrm{~L}_{a}^{-1}}$ is isomorphic to $\mathbb{C}\left[9\right.$, Corollary 1.5]. Every isomorphism $\varphi: A_{\mathrm{R}_{a}^{-1}, \mathrm{~L}_{a}^{-1}} \rightarrow \mathbb{C}$ is also an isomorphism $\varphi: A \rightarrow \mathbb{C}_{\varphi \mathrm{R}_{a} \varphi^{-1}, \varphi \mathrm{L}_{a} \varphi^{-1}}[3$, Lemma 2.5]. Altogether this shows that the set $\left\{\mathbb{C}_{\sigma, \tau} \mid(\sigma, \tau) \in \mathrm{GL}_{\mathbb{R}}(\mathbb{C}) \times \mathrm{GL}_{\mathbb{R}}(\mathbb{C})\right\}$ of all isotopes of $\mathbb{C}$ is dense in $\mathscr{D}_{2}$.

Towards a refinement of this approach to $\mathscr{D}_{2}$ we adopt the following convention. With reference to the standard basis $(1, i)$ of the real vector space $\mathbb{C}$, we identify complex numbers $x_{1}+i x_{2}$ with their coordinate columns $\left(\begin{array}{l}x_{1} \\ x_{2}\end{array}\right)$, and linear operators $\sigma \in \mathrm{GL}_{\mathbb{R}}(\mathbb{C})$ with their matrices $S=(\sigma(1) \sigma(i)) \in \mathrm{GL}(2)$. In particular, complex conjugation and rotation in the complex plane by $\frac{2 \pi}{3}$ are described by the matrices

$$
K=\left(\begin{array}{rr}
1 & 0 \\
0 & -1
\end{array}\right) \quad \text { and } \quad R=\frac{1}{2}\left(\begin{array}{cc}
-1 & -\sqrt{3} \\
\sqrt{3} & -1
\end{array}\right)
$$

respectively. They generate the cyclic group $\mathrm{C}_{2}=\langle K\rangle$ of order 2 and the dihedral group $\mathrm{D}_{3}=\langle R, K\rangle$ of order 6 . Moreover, we denote by $\mathscr{S}_{2}$ the set of all real $2 \times 2$-matrices that are positive definite symmetric and have determinant 1. For each $i \in\{0,1\}$ we set $\mathscr{S}_{2} K^{i}=\left\{S K^{i} \mid S \in \mathscr{S}_{2}\right\}$, and likewise $K^{i} \mathscr{S}_{2}=\left\{K^{i} S \mid S \in \mathscr{S}_{2}\right\}$. Note that $\mathscr{S}_{2} K^{i}=K^{i} \mathscr{S}_{2}$. Finally we denote, for any category $\mathscr{C}$, by $\mathscr{C}(X, Y)$ the morphism set in $\mathscr{C}$ with domain $X$ and codomain $Y$. Now the following holds true [8, Propositions 3.1 and $3.2])$.

Proposition 4.9. (i) The set $\left\{\mathbb{C}_{\sigma, \tau} \mid(\sigma, \tau) \in \bigcup_{(i, j) \in\{0,1\}^{2}}\left(\mathscr{S}_{2} K^{i} \times \mathscr{S}_{2} K^{j}\right)\right\}$ is dense in $\mathscr{D}_{2}$.

(ii) If $(i, j) \in\{(0,0),(0,1),(1,0)\}$ and $(A, B),(C, D) \in \mathscr{S}_{2}^{2}$, then

$$
\mathscr{D}_{2}\left(\mathbb{C}_{A K^{i}, B K^{j}}, \mathbb{C}_{C K^{i}, D K^{j}}\right)=\left\{F \in \mathrm{C}_{2} \mid\left(F A F^{t}, F B F^{t}\right)=(C, D)\right\} .
$$

(iii) If $(A, B),(C, D) \in \mathscr{S}_{2}^{2}$, then

$$
\mathscr{D}_{2}\left(\mathbb{C}_{K A, K B}, \mathbb{C}_{K C, K D}\right)=\left\{F \in \mathrm{D}_{3} \mid\left(F A F^{t}, F B F^{t}\right)=(C, D)\right\} .
$$

The left actions of $\mathrm{C}_{2}$ and $\mathrm{D}_{3}$ on $\mathscr{S}_{2}^{2}$ by simultaneous conjugation give rise to the groupoids $\mathrm{C}_{2} \mathscr{S}_{2}^{2}$ and ${ }_{\mathrm{D}_{3}} \mathscr{S}_{2}^{2}$ respectively. By virtue of Proposition 4.9(ii) we obtain for each $(i, j) \in\{(0,0),(0,1),(1,0)\}$ a full and faithful functor $\mathscr{J}_{i j}:{ }_{\mathrm{C}_{2}} \mathscr{S}_{2}^{2} \rightarrow \mathscr{D}_{2}$, defined on objects by $\mathscr{J}_{i j}(A, B)=\mathbb{C}_{A K^{i}, B K^{j}}$ and on morphisms by $\mathscr{J}_{i j}(F,(A, B),(C, D))=F$. According to Lemma 3.2 
the functor $\mathscr{J}_{i j}: \mathrm{C}_{2} \mathscr{S}_{2}^{2} \rightarrow \mathscr{D}_{2}$ induces a functor $\mathscr{J}_{i j}: \mathrm{C}_{2} \mathscr{S}_{2}^{2} \rightarrow \mathscr{D}_{2}^{(-1)^{j},(-1)^{i}}$ which in fact, due to Proposition 4.9(i) and Proposition 2.2, is dense, and hence an equivalence of categories.

Setting out from Proposition 4.9(iii), we obtain in a similar vein an equivalence of categories $\mathscr{J}_{11}:{ }_{\mathrm{D}_{3}} \mathscr{S}_{2}^{2} \rightarrow \mathscr{D}_{2}^{--}$, defined on objects by $\mathscr{J}_{11}(A, B)=\mathbb{C}_{K A, K B}$ and on morphisms by $\mathscr{J}_{11}(F,(A, B),(C, D))=F$.

Corollary 4.10. The blocks $\mathscr{D}_{2}^{++}, \mathscr{D}_{2}^{+-}$and $\mathscr{D}_{2}^{-+}$are equivalent to each other, but not equivalent to $\mathscr{D}_{2}^{--}$.

Proof. The first statement follows from the equivalences of categories

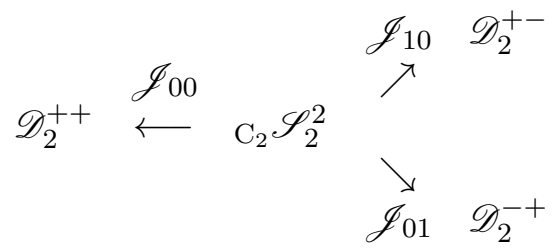

In view of these and the equivalence of categories $\mathscr{J}_{11}: \mathrm{D}_{3} \mathscr{S}_{2}^{2} \rightarrow \mathscr{D}_{2}^{--}$, the second statement is logically equivalent to the categorical inequivalence of ${ }_{\mathrm{D}_{3}} \mathscr{S}_{2}^{2}$ and ${ }_{\mathrm{C}_{2}} \mathscr{S}_{2}^{2}$, which indeed holds true because ${ }_{\mathrm{D}_{3}} \mathscr{S}_{2}^{2}((\mathbb{I}, \mathbb{I}),(\mathbb{I}, \mathbb{I}))=\mathrm{D}_{3}$, while $\left|\mathrm{C}_{2} \mathscr{S}_{2}^{2}((A, B),(A, B))\right| \leq 2$ for all objects $(A, B) \in \mathscr{S}_{2}^{2}$.

Remark 4.11. The inclusion functor of the non-full and dense subcategory $\mathrm{C}_{2} \mathscr{S}_{2}^{2} \subset \mathrm{D}_{3} \mathscr{S}_{2}^{2}$ together with the equivalences $\mathscr{J}_{11}: \mathrm{D}_{3} \mathscr{S}_{2}^{2} \rightarrow \mathscr{D}_{2}^{--}$and $\mathscr{J}_{i j}: \quad \mathrm{C}_{2} \mathscr{S}_{2}^{2} \rightarrow \mathscr{D}_{2}^{(-1)^{j},(-1)^{i}},(i, j) \in\{(0,0),(1,0),(0,1)\}$, yields non-full, faithful and dense functors $\mathscr{D}_{2}^{\alpha \beta} \rightarrow \mathscr{D}_{2}^{--}$for all $(\alpha, \beta) \in\{(1,1),(1,-1)$, $(-1,1)\}$.

\section{References}

[1] A. A. Albert. Non-associative algebras. I. Fundamental concepts and isotopy. Ann. of Math. (2), 43:685-707, 1942.

[2] A. A. Albert. Absolute valued real algebras. Ann. of Math. (2), 48:495$501,1947$.

[3] G. Benkart, D. Britten, and J. M. Osborn. On applications of isotopy to real division algebras. Hadronic J., 4:497-529, 1981.

[4] R. Bott and J. Milnor. On the parallelizability of the spheres. Bull. A. M. S., 64:87-89, 1958.

[5] J. A. Cuenca Mira. On composition and absolute-valued algebras. Proc. Roy. Soc. Edinburgh Sect. A, 136A(4):717-731, 2006.

[6] E. Darpö. Isotopes of Hurwitz algebras. arXiv: 1012.1849, 2010. 
[7] L. E. Dickson. Linear algebras with associativity not assumed. Duke Math. J., 1:113-125, 1935.

[8] E. Dieterich. Classification, automorphism groups and categorical structure of the two-dimensional real division algebras. J. Algebra Appl., 4(5):517-538, 2005.

[9] E. Dieterich and J. Öhman. On the classification of 4-dimensional quadratic division algebras over square-ordered fields. J. London Math. Soc., 65(2):285-302, 2002.

[10] F. G. Frobenius. Über lineare Substitutionen und bilineare Formen. J. Reine Angew. Math., 84:1-63, 1878.

[11] H. Hopf. Ein topologischer Beitrag zur reellen Algebra. Comment. Math. Helv., 13:219-239, 1940/41.

[12] M. Kervaire. Non-parallelizability of the $n$-sphere for $n>7$. Proc. Nat. Acad. Sci., 44:280-283, 1958.

[13] M. Koecher and R. Remmert. The isomorphism theorems of Frobenius, Hopf and Gelfand-Mazur. In Numbers, Graduate Texts in Mathematics, pages 221-247. Springer, 1995.

Erik Darpö Mathematical Institute

24-29 St Giles'

Oxford OX1 3LB

United Kingdom

Ernst Dieterich Matematiska institutionen

Uppsala universitet

Box 480

SE-751 06 Uppsala

Sweden

Erik.Darpo@maths.ox.ac.uk

Ernst.Dieterich@math.uu.se 